\title{
ON THE DECAY OF A VISCOUS VORTEX*
}

\author{
By ROBERT A. GRANGER (U.S. Naval Academy)
}

1. Introduction. In order for a vortex flow to be circulation-preserving, Kelvin [1] stated that the total acceleration vector must admit a potential such that the curl of the acceleration vector vanishes. For inviscid fluids, the circulation within a circuit moving with the fluid cannot change with time. When the flow is steady and when in particular the circulation is an arbitrary function of the stream function, it is trivial to show that the inviscid flow is circulation-preserving. Thus, for inviscid steady vortex flows, if the ratio of the axial velocity to the vorticity about the axis of rotation is constant, the flow is indeed circulation-preserving.

Consider the converse. Suppose experimental measurements revealed that the streamlines and vortex lines were coincident. One might be tempted to identify the physical flow as circulation-preserving. The question arises whether viscous unsteady flows permit the existence of coincident vortex lines and streamlines; i.e., is the ratio of the axial velocity to the vorticity about the axis of rotation constant for, say, a real decaying vortex?

2. Equations of motion. The Navier-Stokes equations for axisymmetric flow of an incompressible fluid medium, expressed in cylindrical coordinates, are

$$
\begin{gathered}
\rho\left(\frac{\partial v_{r}}{\partial t}+v_{r} \frac{\partial v_{r}}{\partial r}+v_{z} \frac{\partial v_{r}}{\partial z}-\frac{v_{\theta}^{2}}{r}\right)=-\frac{\partial p}{\partial r}+\mu\left(\frac{\partial^{2} v_{r}}{\partial r^{2}}+\frac{1}{r} \frac{\partial v_{r}}{\partial r}-\frac{v_{r}}{r^{2}}+\frac{\partial^{2} v_{r}}{\partial z^{2}}\right), \\
\rho\left(\frac{\partial v_{z}}{\partial t}+v_{r} \frac{\partial v_{z}}{\partial r}+v_{z} \frac{\partial v_{z}}{\partial z}\right)=-\frac{\partial p}{\partial z}+\mu\left(\frac{\partial^{2} v_{z}}{\partial r^{2}}+\frac{1}{r} \frac{\partial v_{z}}{\partial r}+\frac{\partial^{2} v_{z}}{\partial z^{2}}\right), \\
\rho\left(\frac{\partial v_{\theta}}{\partial t}+v_{r} \frac{\partial v_{\theta}}{\partial r}+v_{s} \frac{\partial v_{\theta}}{\partial z}+\frac{v_{r} v_{\theta}}{r}\right)=\mu\left(\frac{\partial^{2} v_{\theta}}{\partial r^{2}}+\frac{1}{r} \frac{\partial v_{\theta}}{\partial r}-\frac{v_{\theta}}{r^{2}}+\frac{\partial^{2} v_{\theta}}{\partial z^{2}}\right) .
\end{gathered}
$$

The continuity equation is

$$
(1 / r)\left(\partial\left(r v_{r}\right) / \partial r\right)+\left(\partial v_{z} / \partial z\right)=0 .
$$

It is important to note at this point that, because a fixed nonrotating coordinate system has been chosen, the Coriolis terms do not enter the equations of motion.

The equations of motion are simplified by reducing the number of unknowns. The first and second momentum equations, (1) and (2), are combined to eliminate the pressure. The radial and axial velocity components are expressed in terms of the stream function $\psi$, and the circumferential velocity component is expressed in terms of the circulation $\Gamma$ by defining

$$
v_{r}=\left(Q . / r_{0} l_{\eta}^{1 / 2}\right)(\partial \Psi / \partial \xi),
$$

* Received September 27, 1971; revised version received March 5, 1972. This research was supported by NARC, U.S. Naval Academy, Annapolis, Md. 


$$
\begin{aligned}
& v_{z}=\left(-2 Q_{s} / r_{0}^{2}\right)(\partial \Psi / \partial \eta), \\
& v_{\theta}=\Gamma_{\infty} \bar{\Gamma} / 2 \pi r_{0} \eta^{1 / 2},
\end{aligned}
$$

where the dimensionless dependent variables are defined as

$$
\bar{\Gamma}=\Gamma / \Gamma_{\infty}, \quad \bar{\psi}=\psi / Q_{z} .
$$

Flow parameters that are constant are the sink volume rate of flow $Q_{s}$, the potential circulation $\Gamma_{\infty}$, a reference radial length $r_{0}$, and a reference axial length $l$. The dimensionless independent variables $\eta, \xi$ and $\tau$ are defined as

$$
\eta=\left(r / r_{0}\right)^{2}, \quad \xi=z / l, \quad \tau=4 \nu t / r_{0}^{2} .
$$

If we define

$$
\alpha=\left(r_{0} / l\right)^{2}, \quad N=Q_{s} / \nu l, \quad R_{0}=\left(2 \pi Q_{s} / r_{0} \Gamma_{\infty}\right)
$$

as a dimensionless characteristic length, the axial Reynolds number and the Rossby number, respectively, the equations of motion produced by substituting Eqs. (5)-(10) into the momentum equations resulting from elimination of the pressure are, after algebraic manipulations,

$$
\begin{aligned}
& \frac{1}{8} N R_{0}^{-2} \eta^{-2} \bar{\Gamma} \frac{\partial \bar{\Gamma}}{\partial \xi}=\frac{1}{2} N\left(\frac{\partial \Psi}{\partial \xi} \frac{\partial^{3} \bar{\psi}}{\partial \eta^{3}}-\frac{\partial \Psi}{\partial \eta} \frac{\partial^{3} \bar{\psi}}{\partial \xi \partial \eta^{2}}\right) \\
& +\frac{1}{8} \alpha N \eta^{-2}\left(\eta \frac{\partial \Psi}{\partial \xi} \frac{\partial^{3} \Psi}{\partial \eta \partial \xi^{2}}-\frac{\partial \Psi}{\partial \xi} \frac{\partial^{2} \bar{\psi}}{\partial \xi^{2}}-\eta \frac{\partial \bar{\psi}}{\partial \eta} \frac{\partial^{3} \bar{\psi}}{\partial \xi^{3}}\right) \\
& -\eta \frac{\partial^{4} \Psi}{\partial \eta^{4}}-2 \frac{\partial^{3} \Psi}{\partial \eta^{3}}-\frac{\alpha}{2} \frac{\partial^{4} \Psi}{\partial \eta^{2} \partial \xi^{2}}-\frac{1}{16} \alpha^{2} \eta^{-1} \frac{\partial^{4} \Psi}{\partial \xi^{4}}+\frac{\partial^{3} \Psi}{\partial \tau \partial \eta^{2}}+\frac{1}{4} \alpha \eta^{-1} \frac{\partial^{3} \psi}{\partial \tau \partial \xi^{2}}
\end{aligned}
$$

and

$$
\frac{\partial \bar{\Gamma}}{\partial \tau}+\frac{N}{2}\left(\frac{\partial \bar{\psi}}{\partial \xi} \frac{\partial \bar{\Gamma}}{\partial \eta}-\frac{\partial \Psi}{\partial \eta} \frac{\partial \bar{\Gamma}}{\partial \xi}\right)=\eta \frac{\partial^{2} \bar{\Gamma}}{\partial \eta^{2}}+\frac{\alpha}{4} \frac{\partial^{2} \bar{\Gamma}}{\partial \xi^{2}}
$$

from Eq. (3).

The present theory considers real vortex flows of large axial extent compared to radial extent. For $\alpha \ll 1$, the individual terms of Eqs. (11) and (12) are compared so that Eq. (11) reduces to

$$
\eta^{2}\left[\frac{\partial^{2}}{\partial \eta^{2}}\left(\frac{\partial \Psi}{\partial \tau}-\eta \frac{\partial^{2} \bar{\psi}}{\partial \eta^{2}}\right)+\frac{N}{2}\left(\frac{\partial \bar{\psi}}{\partial \xi} \frac{\partial^{3} \Psi}{\partial \eta^{3}}-\frac{\partial \Psi}{\partial \eta} \frac{\partial^{3} \Psi}{\partial \xi \partial \eta^{2}}\right)\right]=\frac{1}{8} N R_{0}^{-2} \bar{\Gamma} \frac{\partial \bar{\Gamma}}{\partial \xi}
$$

and Eq. (12) reduces to

$$
\frac{\partial \bar{\Gamma}}{\partial \tau}+\frac{N}{2}\left(\frac{\partial \bar{\psi}}{\partial \xi} \frac{\partial \bar{\Gamma}}{\partial \eta}-\frac{\partial \Psi}{\partial \eta} \frac{\partial \bar{\Gamma}}{\partial \xi}\right)=\eta \frac{\partial^{2} \bar{\Gamma}}{\partial \eta^{2}} .
$$

The above equations account for two phenomena of three-dimensional rotational flows: vortex diffusion and vortex stretching. Eq. (13) accounts for vortex stretching through the swirl term $\frac{1}{8} N R_{0}^{-2} \bar{\Gamma}(\partial \bar{\Gamma} / \partial \xi)$ and is absolutely essential for vortex breakdown. The swirl term gives the crucial interaction between the axial and rotational components of motion. Eq. (14) is recognized as the general circulation theorem. 
3. Exact solutions. Solutions of the steady flow form of Eqs. (13) and (14) have been presented by Granger [2] and applied to a specific forced vortex flow. Comparisons of the predictions of the mathematical model with experimental measurements were excellent in the central portion of the vortex flow, i.e. the region removed from the free surface and the bottom surface of the vortex.

Two interesting solutions of Eqs. (13) and (14) exist if one assumes that the circulation is a function of the stream-function. A classic solution of Eqs. (13) and (14) is

$$
\bar{\psi}=-\eta / k \tau, \quad \bar{\Gamma}=\left(a_{1} / k\right) \exp (-\eta / \tau)-1,
$$

due to Oseen [3]. The constants $k$ and $a_{1}$ are evaluated from initial and boundary conditions. The vortex lines corresponding to the flow are helices around the axis of the vortex.

The other interesting solution of Eqs. (13) and (14) can be found by the method of separation of variables and by assuming the solutions harmonic in time. From the definitions of $\bar{\psi}$ and $\bar{\Gamma}$, it is obvious that in order to keep velocities finite on the axis, allowing no steps or kinks in the profile and having a nonrotating coordinate system, it is necessary to have

$$
\left.\frac{\partial \Psi}{\partial \xi}\right|_{\eta=0}=\left.\bar{\Gamma}\right|_{\eta=0}=\left.\frac{\partial v_{z}}{\partial r}\right|_{r=0}=0 .
$$

Fundamental solutions of Eqs. (13) and (14) satisfying the "inner" boundary conditions of Eq. (16) are

$$
\begin{gathered}
\bar{\psi}=2(\beta \eta)^{1 / 2} f(\xi) J_{1}\left[2(\beta \eta)^{1 / 2}\right] \exp (-\beta \tau), \\
\bar{\Gamma}=\frac{1}{2} \beta^{-1 / 2} \bar{\psi},
\end{gathered}
$$

where $\beta=1 / 4 R_{0}$ is the characterizing frequency and $f(\xi)$ is based upon physically admissible expressions at, say, the outer boundary, where $r$ is equal to $r_{0}$. For example, if $f(\xi)=A \xi+B$, the vortex is identified as a Beltrami vortex flow, since the cross product of the vorticity vector with the velocity vector is zero. If $f(\xi)=$ constant, one obtains the results of Caldonazzo [4], who considered a vortex having no radial flow. The latter solution, given by Eq. (18), easily leads to the relation that the ratio of the axial velocity to the vorticity about the axis of rotation is constant. Thus streamlines and vortex lines can be coincident for unsteady viscous vortex flows, and the flow is not circulation-preserving.

\section{References}

[1] Lord Kelvin, On vortex motion in Mathematical and physical papers, Cambridge University Press IV, 1910

[2] R. Granger, A steady axisymmetric vortex flow, Geophys. Fluid Dyn. 3, 45-88 (1972)

[3] C. W. Oseen, Hydromechanik, Ark. f. Math. Astron. och Fys. 7, 82 (1911)

[4] B. Caldonazzo, Un osservazione a proposito di moti viscosi simmetrici rispetto ad un asse, Rend. Acc. Lincei 6, 152 (1927) 